\title{
A Complete Image Compression Scheme Based on Overlapped Block Transform with Post-Processing
}

\author{
C. Kwan, ${ }^{1}$ B. Li, ${ }^{2}$ R. Xu, ${ }^{1}$ X. Li, ${ }^{1}$ T. Tran, ${ }^{3}$ and T. Nguyen ${ }^{4}$ \\ ${ }^{1}$ Intelligent Automation, Inc. (IAI), 15400 Calhoun Drive, Suite 400, Rockville, MD 20855, USA \\ ${ }^{2}$ Department of Computer Science and Engineering, Ira. A. Fulton School of Engineering, Arizona State University, P. O. Box 878809, \\ Tempe, AZ 85287-8809, USA \\ ${ }^{3}$ Department of Electrical and Computer Engineering, The Whiting School of Engineering, The Johns Hopkins University, Baltimore, \\ MD 21218, USA \\ ${ }^{4}$ Department of Electrical and Computer Engineering, Jacobs School of Engineering, University of California, San Diego, La Jolla, \\ CA 92093-0407, USA
}

Received 29 April 2005; Revised 19 December 2005; Accepted 21 January 2006

Recommended for Publication by Dimitrios Tzovaras

A complete system was built for high-performance image compression based on overlapped block transform. Extensive simulations and comparative studies were carried out for still image compression including benchmark images (Lena and Barbara), synthetic aperture radar (SAR) images, and color images. We have achieved consistently better results than three commercial products in the market (a Summus wavelet codec, a baseline JPEG codec, and a JPEG-2000 codec) for most images that we used in this study. Included in the system are two post-processing techniques based on morphological and median filters for enhancing the perceptual quality of the reconstructed images. The proposed system also supports the enhancement of a small region of interest within an image, which is of interest in various applications such as target recognition and medical diagnosis.

Copyright @ 2006 Hindawi Publishing Corporation. All rights reserved.

\section{INTRODUCTION}

The importance of image compression may be illustrated by the following examples. For TV-quality color image that is $512 \times 512$ with 24-bit color, it takes 6 million bits to represent the image. For $14 \times 17$ inch radiograph scanned at 70 micrometer with 12-bit gray scale, it takes about 1200 million bits. If one uses a telephone line with 28,800 baud rate to transmit 1 frame of TV image without compression, it will take 4 minutes, and it will take 11.5 hours to transmit a frame of radiograph. Commonly used image compression approaches such as JPEG use discrete-cosine-transform (DCT)-based transform which introduces annoying block artifacts, especially at high compression ratio, making such approaches undesirable for applications such as target recognition and medical diagnosis.

The main objective in this research is to achieve high compression ratios for still images, such as SAR, and color images, without suffering from the annoying blocking artifacts from a JPEG-like coder (DCT-based) or ringing artifacts from wavelet-based codecs (JPEG-2000, e.g.). We aim at building a complete codec that can provide similar perceptual quality as other algorithms but with a higher compression ratio. Additionally, we also want to provide the flexibility in image transmission with embedded bit streams and the region-of-interest enhancement that is often of interest in many applications.

The objective was achieved mainly by using the overlapped block transform wavelet coder (OBTWC). OBTWC transforms a set of overlapped blocks (e.g., $40 \times 40$ pixels) into $8 \times 8$ blocks in the frequency domain. By using a bank of filters with carefully designed coefficients in performing the image transformation, the coder retains the simplicity of block transform and, at the same time, does not have blocking artifacts in high compression ratios due to the presence of overlapped block transform. Meanwhile, compared with zero-tree wavelet transform, the OBTWC offers more flexibility in frequency spectrum partitioning, higher energy compaction, and parallel processing for fast implementation. OBTWC also maps the transformed image into a multiresolution representation that resembles the zero-tree wavelet transform, and thus embedded stream is a reality. In addition to adopting the OBTWC, we also propose two post-processing techniques that aim at improving the visual 
quality by eliminating some ringing artifacts at very high compression ratio. Reference [1] summarized the application of OBTWC to SAR image compression. However, in [1], we did not give details of our algorithm, the post-processing algorithms, the tool for region-of-interest selection, and compression results of other images.

The rest of the paper is organized as follows. In Section 2, we review the background and theory of OBTWC. Section 3 summarizes our results. The still image compression results include benchmark images (Lena and Barbara), SAR images, and color images. Since degradation in high compression ratio images is unavoidable, two post-processing techniques were developed in this research to enhance the perceptual performance of reconstructed images. A novel technique to enhance a small region of an image was also developed here which could be useful for target recognition. Extensive comparative studies have been carried out with a wavelet coder from commercial market, a baseline JPEG coder (DCT-based), and a JPEG-2000 coder (wavelet-based). Our coder performs consistently better in almost all the images that we used in this study. A computational complexity analysis is also carried out in this section. Finally, Section 4 concludes the paper with some suggestions for future research.

\section{THEORETICAL BACKGROUND ON THE OBTWC ALGORITHM}

\subsection{Background}

Popular image compression schemes such as JPEG [2] use DCT as the core technology. DCT suffers from the blocking artifacts in high compression ratio, and hence it is not suitable for high compression ratio applications. The development of the lapped orthogonal transform [3-5] and its generalized version GenLOT [6, 7] helps to solve the annoying blocking artifact problem to a certain extent by borrowing pixels from the adjacent blocks to produce the transform coefficients of the current block. However, global information has not been taken to its full advantage in most cases, the quantization and the entropy coding of the transform coefficients are still done independently from block to block.

Subband coding has been used in JPEG-2000 thanks to the development of the discrete wavelet transform $[8,9]$. Wavelet representations with implicit overlapping and variable-length basis functions produce smoother and more perceptually pleasant reconstructed images. Moreover, wavelet's multiresolution characteristics have created an intuitive foundation on which simple, yet sophisticated, methods of encoding the transform coefficients are developed.

Instead of aiming for exceptional decorrelation between subbands, current state-of-the-art wavelet coders [10-12] look for other filter properties that still maintain perceptual quality at low bit rates, and then exploit the correlation across the subbands by an elegant combination of scalar quantizers and bit-plane entropy coders. Global information is taken into account at every stage. Nevertheless, in frequency domain, the conventional wavelet transform simply provides an octave-band representation of signals. The conventional dyadic wavelet transform performs a nonuniform $M$-band partition of the frequency spectrum. This may lead to low energy compaction, especially when applying to mediumto high-frequency signals, or signals with well-localized frequency components. In such cases, $M$-channel uniform filter banks may be better alternatives.

From a filter bank viewpoint, the dyadic wavelet transform is simply an octave-band representation for signals; the discrete dyadic wavelet transform can be obtained by iterating on the lowpass output of a PR (perfect reconstruction) two-channel filter bank with enough regularity [13-15]. For a true wavelet decomposition, one iterates on the lowpass output only, whereas for a wavelet-packet decomposition, one may iterate on any output.

Progressive image transmission scheme is perfect for the recent explosion of the World Wide Web. This coding approach first introduced by [10] relies on the fundamental idea that more important information (defined here as what decreases a certain distortion measure the most) should be transmitted first. Assume that the distortion measure is mean-squared error (MSE), the transform is paraunitary, and transform coefficients $c_{i j}$ are transmitted one by one, it can be proven that the mean-squared error decreases by $\left[c_{i j}\right] / N$, where $N$ is the total number of pixels. Therefore, larger coefficients should be transmitted first [16]. If one bit is transmitted at a time, this approach can be generalized to ranking the coefficients by bit planes and the most significant bits are transmitted first [10-12]. The most sophisticated wavelet-based progressive transmission schemes $[11,12]$ result in an embedded bit stream (i.e., it can be truncated at any point by the decoder to yield the best corresponding reconstructed image).

Although the wavelet tree provides an elegant hierarchical data structure which facilitates quantization and entropy coding of the coefficients, the efficiency of the coder heavily depends on the transform's ability in generating "enough" zero trees. For nonsmooth images (such as SAR image) that contain a lot of texture and edges, wavelet-based zero tree algorithms are not efficient. As will be seen shortly, our proposed OBTWC shown in Figure 1 is a lot better in terms of achieving higher compression ratio while retaining the same perceptual image quality.

\subsection{Theory of OBTWC}

The theory of lattice structures and design methods for the two-channel filter banks are well established [13, 17]. It is shown in [13] that linear-phase and paraunitary properties cannot be simultaneously imposed on two-channel filter banks, unless for the special case of Haar wavelets. However, when more channels are allowed in the systems, both of the above properties can coexist [13]. For instance, the DCT (discrete cosine transform) and LOT (lapped orthogonal transform) are two examples where both the analysis and synthesis filters $H_{k}(z)$ and $F_{k}(z)$ are linear-phase FIR filters and the corresponding filter banks are paraunitary. In this section, the lattice structure of the $M$-channel linear-phase 


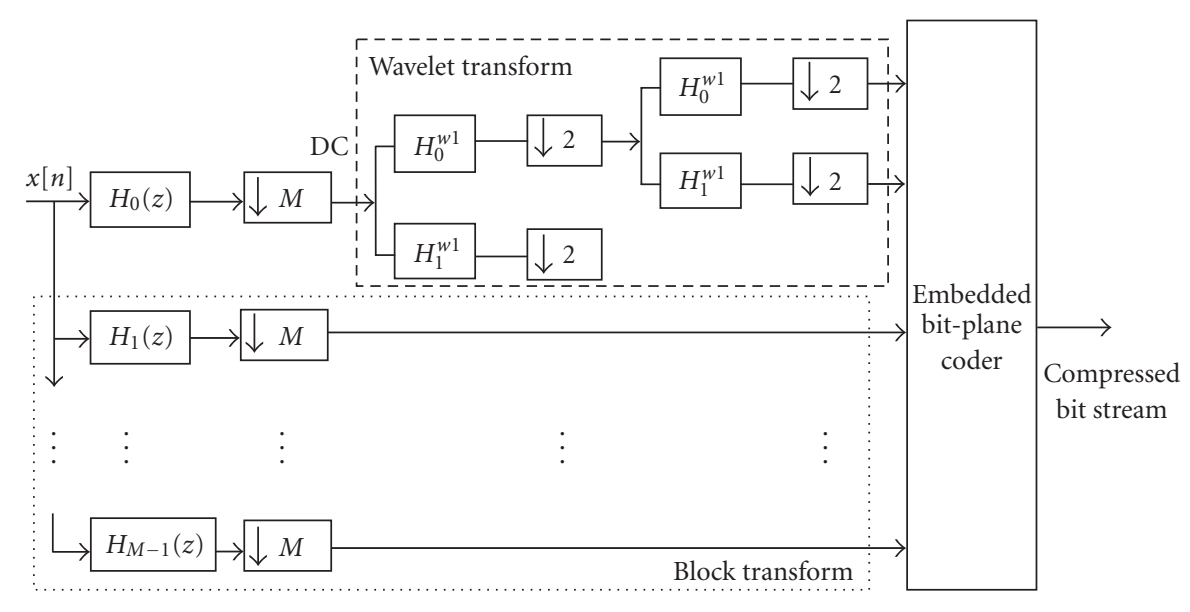

Figure 1: Proposed OBTWC.

paraunitary filter bank (OBTWC) is discussed. It is assumed that the number of channels $M$ is even and the filter length $L$ is a multiple of $M$, that is, $L=N M$.

It is shown in [6] that $M / 2$ filters (in analysis or synthesis) have symmetric impulse responses and the other $M / 2$ filters have antisymmetric impulse responses. Under the assumptions on $N, M$, and on the filter symmetry, the polyphase transfer matrix $H_{p}(z)$ of a linear-phase paraunitary filter bank of degree $N-1$ can be decomposed as a product of orthogonal factors and delays [6], that is,

$$
H_{p}(z)=S Q T_{N-1} \Lambda(z) T_{N-2} \Lambda \cdots \Lambda(z) T_{0} Q
$$

where

$$
\begin{gathered}
Q=\left[\begin{array}{ll}
I & 0 \\
0 & J
\end{array}\right], \quad \Lambda(z)=\left[\begin{array}{cc}
I & 0 \\
0 & z^{-1} I
\end{array}\right], \\
S=\frac{1}{\sqrt{2}}\left[\begin{array}{cc}
S_{0} & 0 \\
0 & S_{1}
\end{array}\right]\left[\begin{array}{cc}
I & J \\
I & -J
\end{array}\right] .
\end{gathered}
$$

Here $I$ and $J$ are the identity and reversed matrices, respectively. $S_{0}$ and $S_{1}$ can be any $M / 2 \times M / 2$ orthogonal matrices and $T_{i}$ are $M \times M$ orthogonal matrices

$$
T_{i}=\left[\begin{array}{cc}
I & I \\
I & -I
\end{array}\right]\left[\begin{array}{cc}
U_{i} & 0 \\
0 & V_{i}
\end{array}\right]\left[\begin{array}{cc}
I & I \\
I & -I
\end{array}\right]=W \Phi_{i} W
$$

where $U_{i}$ and $V_{i}$ are arbitrary orthogonal matrices. The factorization [17] covers all linear-phase paraunitary filter banks with an even number of channels. In other words, given any collection of filters $H_{k}(z)$ that comprise such a filter bank, one can obtain the corresponding matrices $S, Q$, and $T_{k}(z)$. The synthesis procedure is given in [6]. The building blocks in [17] can be rearranged into a modular form where both the DCT and LOT are special cases [6],

$$
\begin{aligned}
& H_{p}(z)=K_{N-1}(z) K_{N-2}(z) \cdots K_{1}(z) K_{0}, \\
& \text { where } \quad K_{i}(z)=\Phi_{i} W \Lambda(z) W .
\end{aligned}
$$

The class of OBTWCs, defined in this way, allows us to view the DCT and LOT as special cases, respectively, for $N=1$ and
$N=2$. The degrees of freedom reside in the matrices $U_{i}$ and $V_{i}$ which are only restricted to be real $M / 2 \times M / 2$ orthogonal matrices. Similar to the lattice factorization in (1), the factorization in (4) is a general factorization that covers all linear-phase paraunitary filter banks with $M$ even and length $L=M N$.

Based on our analysis, there still exists correlation between DC coefficients. To decorrelate the DC band even more, several levels of wavelet decomposition can be used depending on the input image size. Besides the obvious increase in the coding efficiency of DC coefficients thanks to deeper coefficient trees, wavelets provide variably longer bases for the signal's DC component, leading to smoother reconstructed images, that is, blocking artifacts are further reduced. Regularity objective can be added in the transform design process to produce $M$-band wavelets, and a waveletlike iteration can be carried out using uniform-band transforms as well.

The complete proposed coder diagram is depicted in Figure 1. It is a hybrid combination of block transform and wavelet transform. The waveform transform is used for the DC band and overlapped block transforms are used for other bands. The advantage is the enhanced capability of capturing and separating the localized signal components in the frequency domain.

\subsection{Determination of block transform coefficients}

The filter coefficients in $H_{i}(z)$ of Figure 1 require very careful design. We use the following well-known guidelines for filter coefficients to produce a good perceptual image codec.

(i) The filter coefficients should be smooth and symmetric (or antisymmetric). Smoothness controls the noise in a region with constant background. Symmetry allows the use of symmetric extension to process the image's borders.

(ii) They should decay to zero smoothly at both ends. Nonsmoothness at the ends causes discontinuity between 
blocks when the image is compressed. This blocking artifact is typical in JPEC because the DCT coefficients are not smooth at the ends.

(iii) The bandpass and highpass filters should have no DC leakage. Higher-frequency bands will be quantized severely. It is desirable for the lowpass band to contain all of the DC information. Otherwise, if the bandpass and highpass responses to $\omega=0$ are not zero, we see the checkerboard artifact.

(iv) The coefficients should be chosen to maximize coding gain. The coding gain is an approximate measure of energy compaction. A higher gain means higher energy compaction.

(v) Their lengths should be reasonably short to avoid excessive ringing and reasonably long to avoid blocking.

(vi) In the frequency range $|\omega| \leq \pi / M$, the bandpass and highpass responses should be small. This minimizes the quantization effect on bandpass and highpass filters.

To satisfy the above properties, we used an optimization technique. The cost function is a weighted linear combination of coding gain, DC leakage, attenuation around mirror frequencies, and stopband attenuation. It is defined as

$$
\begin{aligned}
C_{\text {overall }}= & k_{1} C_{\text {coding gain }}+k_{2} C_{\mathrm{DC}}+k_{3} C_{\text {mirror }} \\
& +k_{4} C_{\text {analysis stopband }}+k_{5} C_{\text {synthesis stopband }}
\end{aligned}
$$

with $k_{i}$ the weighting factors.

The coding gain cost function is defined as

$$
C_{\text {coding gain }}=10 \log \frac{\sigma_{x}^{2}}{\left(\prod_{k=0}^{M-1} \sigma_{x i}^{2}\left\|f_{i}\right\|^{2}\right)^{1 / M}},
$$

where $\sigma_{x}^{2}$ is the variance of the input signal, $\sigma_{x i}^{2}$ is the variance of the $i$ th subband, and $\left\|f_{i}\right\|^{2}$ is the norm of the $i$ th synthesis filter.

The DC leakage cost function measures the amount of DC energy that leaks out to the bandpass and highpass subbands. The main idea is to concentrate all signal energy at DC into the DC coefficients. This proves to be advantageous in both signal decorrelation and in the prevention of discontinuities in the reconstructed signals. Low DC leakage can prevent the annoying checkerboard artifact that usually occurs when high-frequency bands are severely quantized. The DC cost function is defined as

$$
C_{\mathrm{DC}}=\sum_{i=1}^{M-1} \sum_{n=0}^{L-1} h_{i}(n)
$$

The mirror frequency cost function is a generalization of $C_{\mathrm{DC}}$. Frequency attenuation at mirror frequencies is important in the further reduction of blocking artifacts. The corresponding cost function is

$$
C_{\text {mirror }}=\sum_{i=0}^{M-1}\left|H_{i}\left(e^{j \omega_{m}}\right)\right|^{2}, \quad \omega_{m}=\frac{2 \pi m}{M}, \quad 1 \leq m \leq \frac{M}{2} .
$$

Stopband attenuation criterion measures the sum of all of the filters' energy outside the designated passbands. Mathematically,

$$
\begin{aligned}
& C_{\text {analysis stopband }}=\sum_{i=0}^{M-1} \int_{\omega \in \Omega_{\text {stopband }}} W_{i}^{a}\left(e^{j \omega}\right)\left|H_{i}\left(e^{j \omega}\right)\right|^{2} d \omega, \\
& C_{\text {synthesis stopband }}=\sum_{i=0}^{M-1} \int_{\omega \in \Omega_{\text {stopband }}} W_{i}^{s}\left(e^{j \omega}\right)\left|F_{i}\left(e^{j \omega}\right)\right|^{2} d \omega .
\end{aligned}
$$

In the analysis bank, the stopband attenuation cost helps in improving the signal decorrelation and decreasing the amount of aliasing. In meaningful images, we know a priori that most of the energy is concentrated in low-frequency region. Hence, high stopband attenuation in this part of the frequency spectrum becomes extremely desirable. In the synthesis bank, the reverse is true. Synthesis filters covering lowfrequency bands need to have high stopband attenuation near and/or at $\omega=\pi$ to enhance their smoothness. The biased weighting can be enforced using two simple linear functions $W_{i}^{a}\left(e^{j \omega}\right)$ and $W_{i}^{s}\left(e^{j \omega}\right)$.

The optimization of cost function in (5) is performed by using a nonlinear optimization routine called Simplex in MATLAB. The results are the optimized filter coefficients.

\subsection{Comparison summary between OBTWC, DCT, and wavelet}

Consumers and manufacturers are pushing for higher and higher number of pixels in digital cameras, camcorders, and high-definition TVs. All these advancements call for stringent demands for faster and nicer compression codecs. It will be ideal for a codec to have fast compression and, at the same time, achieves very satisfactory perceptual quality and signalto-noise ratio. The proposed OBTWC has exactly these qualities.

Table 1 summarizes the comparison between three codecs. It can be seen that the proposed codec has more advantages than DCT and wavelet. It is the balanced quality between computational speed and performance that makes the proposed OBTWC stands out among the other codecs.

\subsection{Implementation of a complete coder}

The proposed method was implemented by replacing the transform of an H.263+ codec by the GenLOT transform (using only the I-frame mode for still image compression), with appropriate coefficient reordering. The entropy coding and other parts of the codec are kept the same.

\section{STILL IMAGE COMPRESSION}

Although the component technologies of OBTWC for still image compression were developed before this research, this is the first time that we applied the software to SAR images, and color images. Extensive comparative studies with two commercial products have been carried out in this research. 
TABLE 1: Comparison of different codecs.

\begin{tabular}{|c|c|c|c|}
\hline Performance metrics & $\begin{array}{c}\text { DCT } \\
\text { (core technology in standards } \\
\text { such as JPEG, MPEG, } \\
\text { H263, etc.) }\end{array}$ & $\begin{array}{c}\text { Wavelet } \\
\text { (zero-tree dyadic wavelet } \\
\text { transform and core } \\
\text { technology of JPEG-2000) }\end{array}$ & $\begin{array}{c}\text { OBTWC } \\
\text { (proposed overlapped } \\
\text { block transform } \\
\text { wavelet coder) }\end{array}$ \\
\hline Transmits most important information first & & $\checkmark$ & $\checkmark$ \\
\hline $\begin{array}{l}\text { Simplicity of block transform } \\
\text { (less memory required) }\end{array}$ & $\checkmark$ & & $\checkmark$ \\
\hline $\begin{array}{l}\text { Encodes the whole frame } \\
\text { (larger on-board memory) }\end{array}$ & & $\checkmark$ & \\
\hline $\begin{array}{l}\text { Block artifacts } \\
\text { (lose details in high compression ratio) }\end{array}$ & $\checkmark$ & & \\
\hline Better performance (than DCT) & & $\checkmark$ & $\checkmark$ \\
\hline More computations (than DCT) & & $\checkmark$ & $\checkmark$ \\
\hline Ringing effect & & $\checkmark$ & $\checkmark$ \\
\hline $\begin{array}{l}\text { Flexibility in frequency spectrum partitioning } \\
\text { and higher energy compaction }\end{array}$ & & & $\checkmark$ \\
\hline $\begin{array}{l}\text { Capture and separate localized signal } \\
\text { components in the frequency domain }\end{array}$ & & & $\checkmark$ \\
\hline $\begin{array}{l}\text { Produces smoother and more perceptually } \\
\text { pleasant reconstructed images }\end{array}$ & & $\checkmark$ & $\checkmark$ \\
\hline $\begin{array}{l}\text { Enhances the compression ratio of existing } \\
\text { techniques without sacrificing too much } \\
\text { of the performance/perceptual quality }\end{array}$ & & $\checkmark$ & $\checkmark$ \\
\hline $\begin{array}{l}\text { Texture preservation } \\
\text { (suitable for SAR compression) }\end{array}$ & & & $\checkmark$ \\
\hline $\begin{array}{l}\text { Reversible integer GenLOT available whereas the } \\
\text { standard codec does not allow reversible integer } \\
\text { transform (useful for mobile communications) }\end{array}$ & & & $\checkmark$ \\
\hline Parallel processing capability & $\checkmark$ & & $\checkmark$ \\
\hline
\end{tabular}

In terms of military applications, one can directly apply our still image compression algorithm for image storage and archiving.

\subsection{Benchmark images compression}

In this section, we summarize the application of several progression transmission codecs, including SPIHT (waveletbased method), JPEG, JPEG-2000, and our OBTWC. Benchmark images (Lena and Barbara) were used in this comparative study.

The objective performance criterion we used is called peak signal-to-noise ratio (PSNR) which is defined as

$$
\text { PSNR }=10 \log \frac{255^{2}}{(1 / M) \sum_{n=1}^{M}\left(o_{n}-r_{n}\right)^{2}},
$$

where $o_{n}$ is the $n$th pixel in the original image and $r_{n}$ is the $n$th pixel in the reconstructed image. This is a popular objective method to measure distortion in image compression
TABLE 2: Coding results of various progressive coders for Lena.

\begin{tabular}{l|cccc}
\hline Lena & \multicolumn{4}{|c}{ Progressive transmission coders } \\
\hline Comp. ratio & SPIHT $(9-7 \mathrm{WL})$ & JPEG & JPEG-2000 & OBTWC \\
\hline $1: 8$ & 40.41 & 39.91 & 40.32 & $\mathbf{4 0 . 4 3}$ \\
$1: 16$ & 37.21 & 36.38 & 37.27 & $\mathbf{3 7 . 3 2}$ \\
$1: 32$ & 34.11 & 32.90 & 34.14 & $\mathbf{3 4 . 2 3}$ \\
$1: 64$ & 31.10 & 29.67 & 31.00 & $\mathbf{3 1 . 1 6}$ \\
$1: 100$ & $\mathbf{2 9 . 3 5}$ & 27.80 & 29.12 & 29.31 \\
$1: 128$ & $\mathbf{2 8 . 3 8}$ & 26.91 & 28.00 & 28.35 \\
\hline
\end{tabular}

applications. The higher the PSNR is, the better the compression and decompression performance is.

Table 2 summarizes the PSNR of Lena and Figure 2 depicts the PSNRs of different codecs at different compression ratios. It can be seen that our codec performed consistently better, except in two cases, than other codecs. 


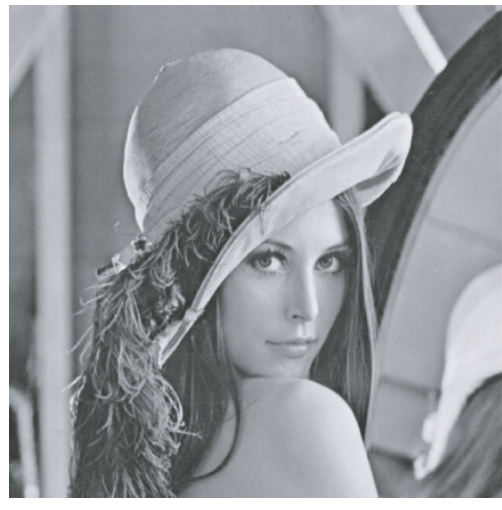

(a)

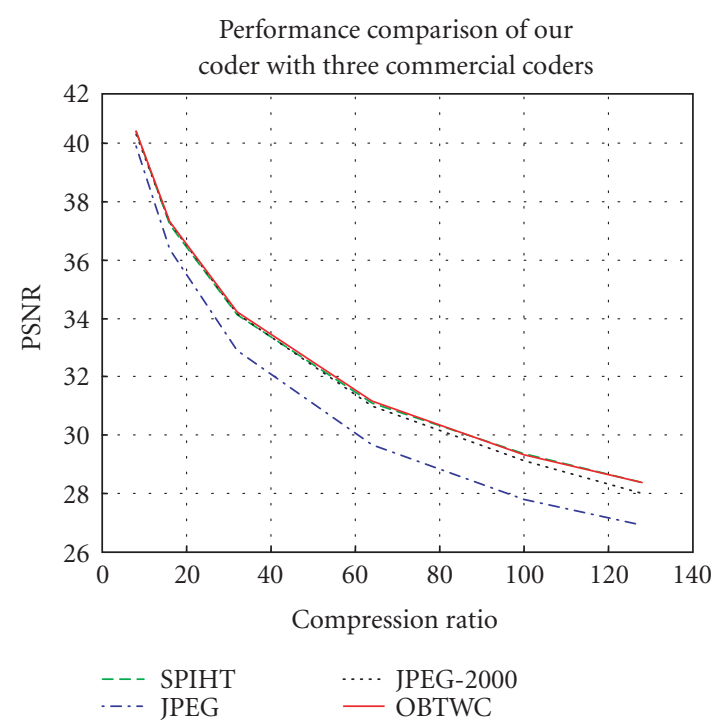

(b)

FIgURE 2: PSNRs of various codecs at different compression ratios for Lena.

TABLE 3: Coding results of various progressive coders for Barbara.

\begin{tabular}{l|cccc}
\hline Barbara & \multicolumn{4}{|c}{ Progressive transmission coders } \\
\hline Comp. ratio & SPIHT $(9-7 \mathrm{WL})$ & JPEG & JPEG 2000 & OBTWC \\
\hline $1: 8$ & 36.41 & 36.31 & 37.17 & $\mathbf{3 8 . 0 8}$ \\
$1: 16$ & 31.40 & 31.11 & 32.29 & $\mathbf{3 3 . 4 7}$ \\
$1: 32$ & 27.58 & 27.28 & 28.39 & $\mathbf{2 9 . 5 3}$ \\
$1: 64$ & 24.86 & 24.58 & 25.42 & $\mathbf{2 6 . 3 7}$ \\
$1: 100$ & 23.76 & 23.42 & 24.06 & $\mathbf{2 4 . 9 5}$ \\
$1: 128$ & 23.35 & 22.68 & 23.37 & $\mathbf{2 4 . 0 1}$ \\
\hline
\end{tabular}

Similarly, Table 3 and Figure 3 summarize the PSNRs for Barbara. Again, our proposed codec performed consistently better than all other codecs.

\subsection{SAR image compression}

We have compressed four types of SAR images: two types from the Air Force, one type from the Army, and one type from NASA. Our algorithm outperforms both wavelet and JPEG coders. The wavelet coder was developed by Summus, Inc. We purchased one copy. It was claimed by Summus that its coder is better than JPEG and other wavelet-based coders. The baseline JPEG coder is a shareware from the Internet. The web address is http://www.geocities.com/SiliconValley/ $7726 /$.

\subsubsection{Air Force cluttered SAR image}

The SAR image (size: $512 \times 480$, gray scale: 8 bits/pixel) was supplied by Air Force Wright Patterson Laboratory (Marvin Soraya). We applied four algorithms to it: our OBTWC algorithm, Summus wavelet coder, JPEG-2000, and JPEG. Three compression ratios were tried. The perceptual differences between the various coders are hard to discern by human eyes. However, the objective performance index (PSNR) tells a big difference. The PSNR is summarized in Table 4. We also plotted PSNRs versus compression ratios. As shown in Figure 4, although our coder has comparable performance as the commercial products, in terms of computational complexity, our algorithm allows parallel processing and hence is much more efficient than other codecs.

\subsubsection{Army's SAR image}

The SAR image (size: $764 \times 764$, gray scale: 8 bits/pixel) was supplied by Army Research Laboratory in Fort Monmouth. Again, four algorithms were applied and the performance is summarized in Table 5. The PSNRs were also plotted against the compression ratios (Figure 5). From Table 5, one can see that our codec is slightly inferior to JPEG-2000 but much better than the other two. But from practical implementation perspective, our codec is much simpler and hence will offer significant advantage for large images such as high-definition TV images.

\subsubsection{NASA's SAR image}

Spaceborne imaging radar-C/X-band synthetic aperture radar (SIR-C/X-SAR) is a joint US-German-Italian Project that uses a highly sophisticated imaging radar to capture images of Earth that are useful to scientists across a great range of disciplines. The instrument was flown on two flights in 1994. One was on space shuttle Endeavor on mission STS-59 April 9-20, 1994. The second flight was on shuttle Endeavor on STS-68 September 30-October 11, 1994. 


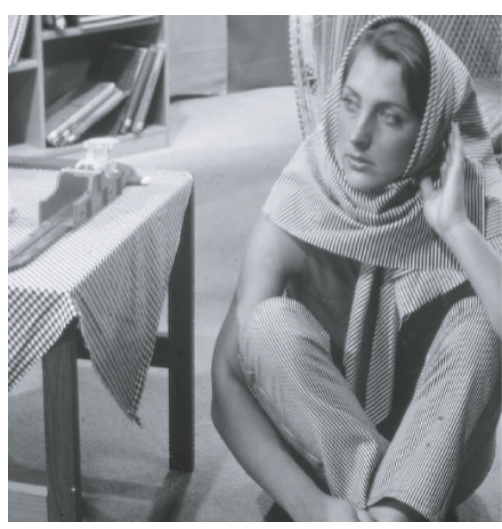

(a)

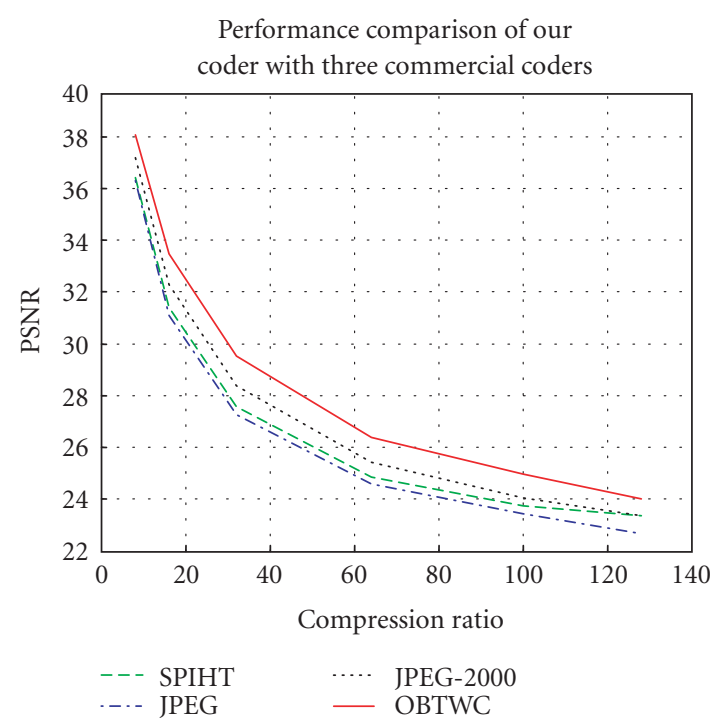

(b)

FIGURE 3: PSNRs of various codecs at different compression ratios for Barbra.

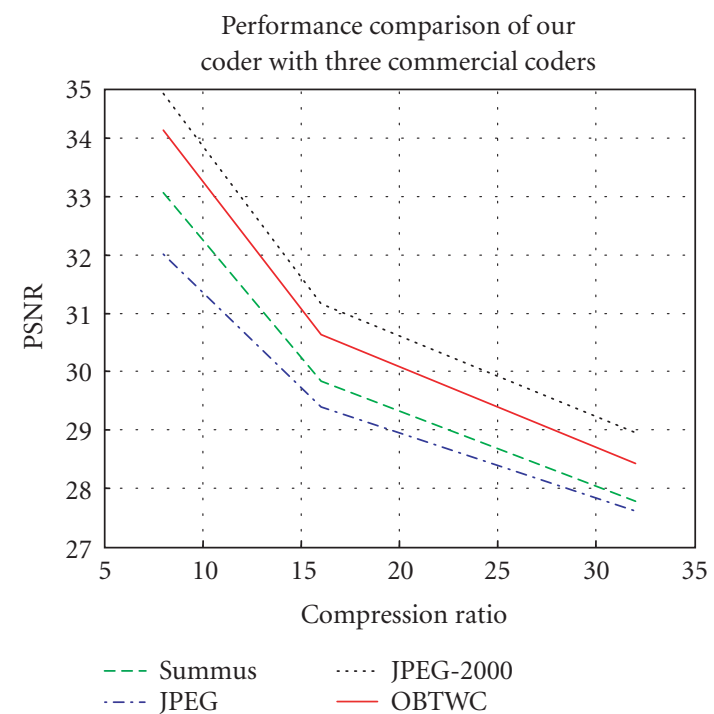

FIGURE 4: PSNR of four compression methods.

The image (size: $945 \times 833$, color depth: 8 bits/pixel) shown in Figure 6 was a recently released image from the SIR-C/X-SAR Project. We applied OBTWC, Summus, JPEG2000, and JPEG codecs to it. The results are summarized in Table 6. The PSNRs versus compression ratios are plotted besides Table 6. Except the 32:1 compression ratio case, our OBTWC outperforms the other codecs in the other two categories. Even in the $32: 1$ case, the OBTWC is only $0.01 \mathrm{~dB}$ less than the wavelet coder is. The plots in Figure 7 show the PSNRs of the three codecs. The OBTWC and Summus have similar performance in this case.
TABLE 4: Performance comparison of our codec with 3 commercial codecs for the Air Force SAR image.

\begin{tabular}{l|cccc}
\hline $\begin{array}{l}\text { Algorithm } \backslash \\
\text { compression ratio }\end{array}$ & OBTWC & Summus & JPEG & JPEG-2000 \\
\hline 8 & 34.14 & 33.06 & 32.02 & $\mathbf{3 4 . 7 7}$ \\
16 & 30.64 & 29.83 & 29.40 & $\mathbf{3 1 . 1 6}$ \\
32 & 28.42 & 27.78 & 27.61 & $\mathbf{2 8 . 9 5}$ \\
\hline
\end{tabular}

TABle 5: Performance comparison of our codec with three commercial codecs for an Army SAR image.

\begin{tabular}{l|rccc}
\hline $\begin{array}{l}\text { Algorithm } \backslash \\
\text { compression ratio }\end{array}$ & OBTWC & Summus & JPEG & JPEG-2000 \\
\hline 8 & 38.07 & 36.73 & 36.32 & $\mathbf{3 9 . 4 1}$ \\
16 & 35.05 & 33.90 & 33.57 & $\mathbf{3 6 . 0 2}$ \\
32 & 32.52 & 31.84 & 31.21 & $\mathbf{3 3 . 0 2}$ \\
\hline
\end{tabular}

\subsection{Color image compression}

We were given four unclassified color images with the size of $344 \times 244$ and YUV (4:4:4) from the Wright Patterson Air Force Laboratory, USA (http://www.wpafb.af.mil). The first image is picture of $2 s 1$ tank. The second is T62 tank. The third is Zill31 armored car. The fourth one is Btr60 armored car. Our OBTWC codec achieved better results in almost all cases except $2 \mathrm{~s} 1$ image. Table 7 summarizes the objective performance of three coders under three different compression ratios. Plots of PSNRs versus the compression ratios are shown in Figure 8. 


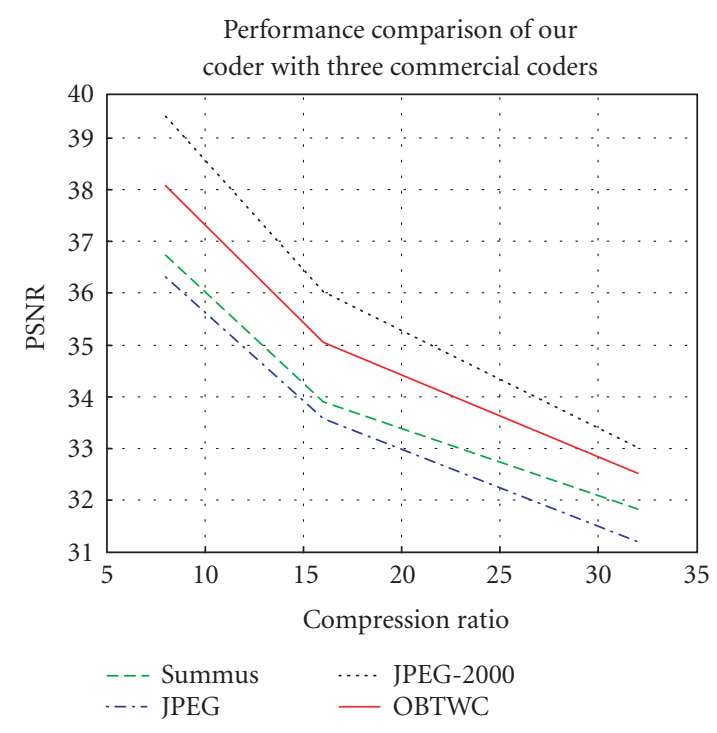

FIGURE 5: PSNRs of four codecs.

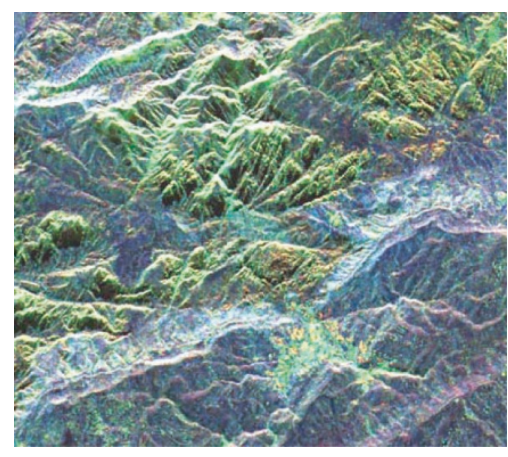

FIgURE 6: Raw image from NASA.

\subsection{Image enhancement of reconstructed images}

The ringing effects in reconstructed images with high compression ratios are caused by the long filter lengths in OBTWC. Although the ringing effect here is less significant than wavelet coders are, it is still an annoying artifact that affects the visual perception of a reconstructed image. Here we propose two approaches to minimize the ringing artifacts. It is worth to mention that image enhancement is performed at the receiving end, and hence this post-processing will not affect the transmission speed.

\subsubsection{Post-processing using nonlinear morphological filters}

The key idea underlying the deringing algorithm is to avoid filtering the entire image blindly, but instead to identify the regions contaminated by ringing and apply the nonlinear smoothing filter only to these regions. As such, the algorithm is a signal-dependent (spatially varying) technique which requires the extraction of certain parameters from the input
TABLE 6: Compression performance of 4 codecs to NASA SAR image.

\begin{tabular}{l|cccc}
\hline $\begin{array}{l}\text { Algorithm } \backslash \\
\text { compression ratio }\end{array}$ & OBTWC & Summus & JPEG & JPEG-2000 \\
\hline 8 & 27.44 & 27.25 & 26.07 & $\mathbf{2 7 . 8 7}$ \\
16 & $\mathbf{2 4 . 5 8}$ & 24.51 & 23.22 & 24.44 \\
32 & 22.40 & $\mathbf{2 2 . 4 1}$ & 21.71 & 22.17 \\
\hline
\end{tabular}

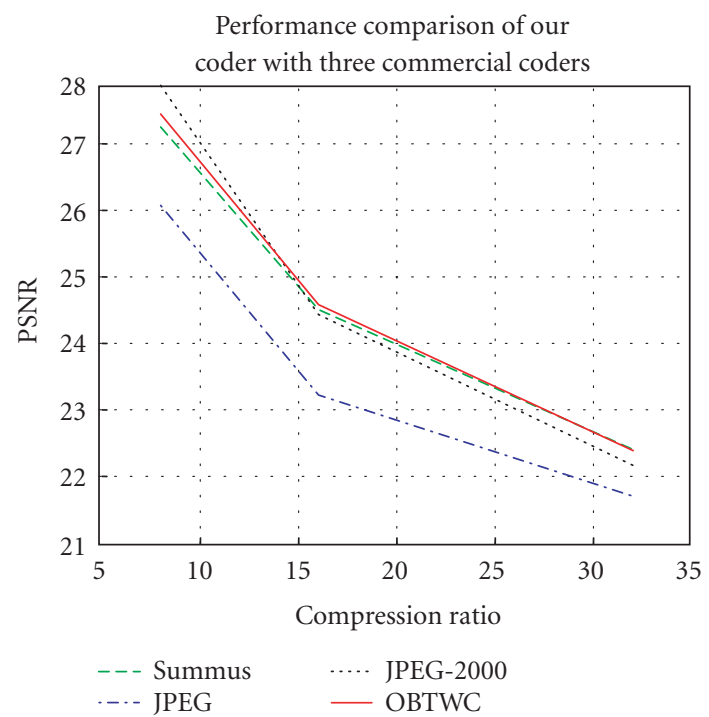

FIgURE 7: PSNRs of four codecs.

image. The choice of a morphological smoothing operator was due to its fit to the purpose and also its very low computational complexity.

\section{Edge detection}

Since the ringing artifact is known to be associated with step edges, the algorithm starts with an edge detection process on the input image. In case of compressed images, the edge detection process is even further complicated because of the blur (associated with compression) which typically causes false negatives (undetected edges) and also the ringing artifact ripples which typically cause false positives (false edges). Consequently, we designed a 3-phase edge detection algorithm in which the following hold.

(1) The first phase is a baseline edge detection algorithm employing Sobel edge detection operator $(5 \times 5)$. The associated threshold for this baseline algorithm is extracted from the input image by paying attention to the ringing around the step edges so that to the binary edge map, only a very little amount of noise due to ringing ripples penetrates.

(2) In spite of the careful threshold selection of the first step, most of the time we still end up with some noise 
TABLE 7: Summary of comparative studies for color images.

\begin{tabular}{|c|c|c|c|c|c|c|c|c|c|c|c|c|}
\hline $\begin{array}{l}\text { Images } \backslash \\
\text { PSNR }\end{array}$ & $\begin{array}{l}\text { JPEG } \\
32: 1\end{array}$ & $\begin{array}{c}\text { Summus } \\
32: 1 \\
32: 1\end{array}$ & $\begin{array}{l}\text { JPEG- } \\
2000\end{array}$ & $\begin{array}{l}\text { OBTWC } \\
32: 1\end{array}$ & $\begin{array}{l}\text { JPEG } \\
64: 1 \\
64: 1\end{array}$ & $\begin{array}{c}\text { Summus } \\
64: 1\end{array}$ & $\begin{array}{l}\text { JPEG- } \\
2000\end{array}$ & $\begin{array}{c}\text { OBTWC } \\
64: 1 \\
100: 1\end{array}$ & $\begin{array}{l}\text { JPEG } \\
100: 1\end{array}$ & $\begin{array}{c}\text { Summus } \\
100: 1\end{array}$ & $\begin{array}{l}\text { JPEG- } \\
2000\end{array}$ & $\begin{array}{l}\text { OBTWC } \\
100: 1\end{array}$ \\
\hline $2 \mathrm{~s} 1$ & 31.56 & 32.44 & 31.96 & 32.18 & 28.78 & 29.67 & 28.77 & 29.52 & 26.64 & 28.18 & 27.15 & 28.20 \\
\hline T62 & 28.45 & 29.07 & 28.70 & 30.05 & 25.37 & 26.37 & 25.72 & 27.15 & 23.45 & 24.97 & 24.24 & 25.61 \\
\hline Zil131 & 28.33 & 29.15 & 28.56 & 30.03 & 25.36 & 26.27 & 25.47 & 26.99 & 23.44 & 24.87 & 23.94 & 25.42 \\
\hline Btr60 & 30.48 & 29.07 & 31.75 & 32.63 & 27.93 & 26.37 & 28.70 & 29.79 & 26.22 & 24.97 & 26.87 & 28.32 \\
\hline
\end{tabular}

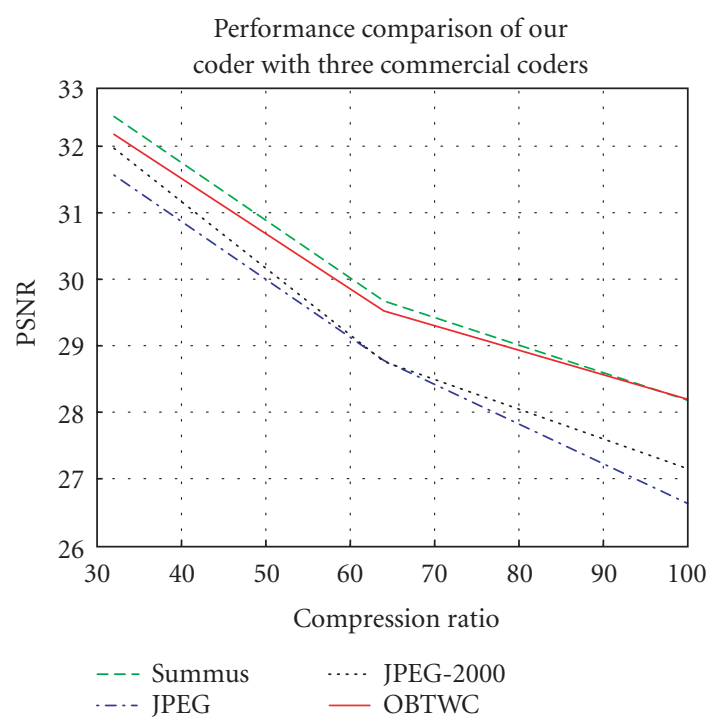

(a) $2 \mathrm{~s} 1$

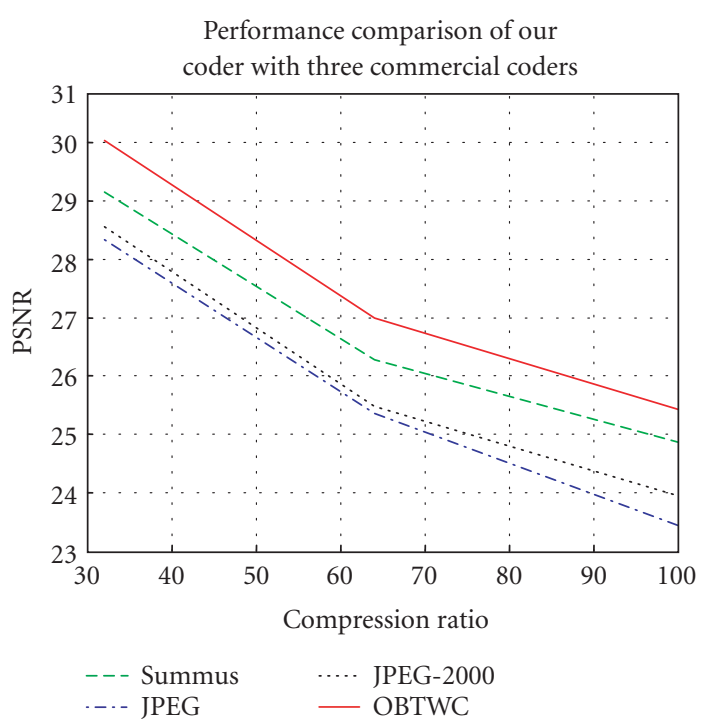

(c) Btr60

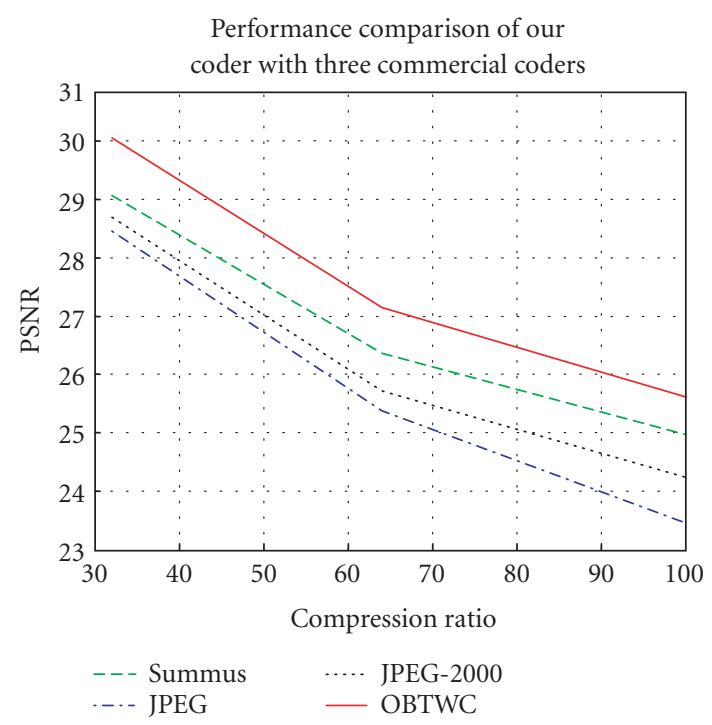

(b) T62

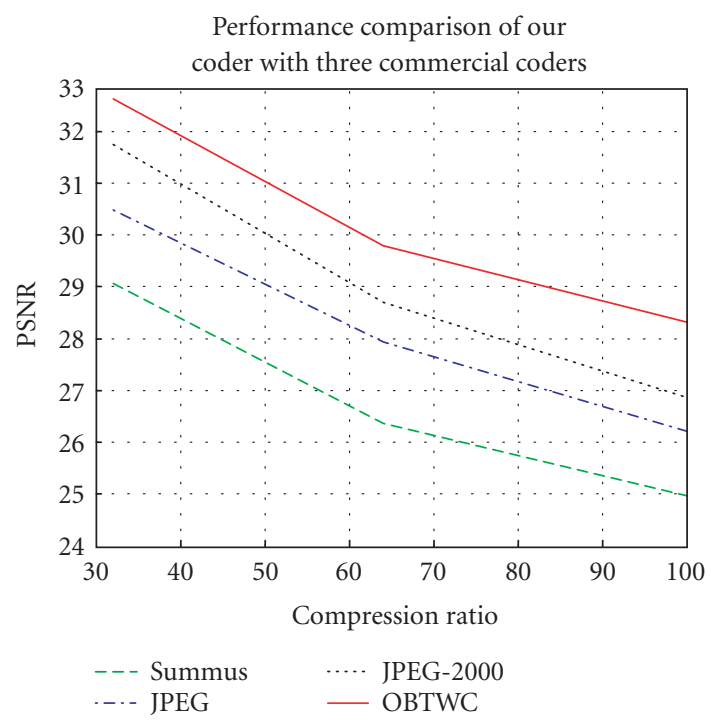

(d) Zil131

FIgURE 8: PSNRs of three codecs for the four color images.

in the binary edge map. To clean this noise, we use a morphological filter consisting of some pruning and hit-or-miss operations.

(3) The cleaned edge map typically has significant discontinuities along many of its edge traces. In this case through a high-level processing, these edge discontinuities are eliminated by edge tracking and linking. As a result, we have a binary edge map which is much improved as compared to the raw output from the first step. 


\section{Edge mask}

The second major step is the generation of the so-called "edge mask." This phase is carried out essentially by a binary closing operation $(3 \times 3)$ on the output of the edge detection phase. The edge mask serves the very important purpose of protecting many genuine image features and high-frequency details such as edges with narrow pulse-like profiles and texture from being destroyed by the consequent morphological smoothing operation.

\section{Filtering mask}

The third major phase is the generation of the so-called "filtering mask." This phase is carried out by a dilation operation $(3 \times 3)$ on the output of the edge detection phase (to isotropically mark the regions surrounding the edges where we know that only these regions are subject to being contaminated with ringing) and then an exclusive-OR operation between the dilation result and the edge mask (output of the second phase) which will remove the regions covered by the edge mask from the filtering mask so that the regions covered by the edge mask will not be filtered. This sequence of operations generates the so-called raw filtering mask. One major feature of the algorithm is that it is employing human visual system (HVS) properties to further process the raw filtering mask and eliminate from it those regions which because of their content and also the masking properties of HVS will not reveal the ringing noise confined to their boundaries. For example, textured regions which could not be identified because of blur in the edge detection step, and therefore not protected by the edge mask, will typically be detected during this phase and consequently removed from the raw filtering mask. The above-mentioned upper local variance limit attributable to ringing ripples is a signal-dependent quantity as well as its dependence on the compression level and we handle it in the appropriate way and extract it from the image in a spatially adaptive way. Once the HVS-based modification is performed on the raw filtering mask, we have the so-called final filtering mask or shortly the filtering mask.

\section{Morphological smoothing}

The fourth major phase of the algorithm is the morphological smoothing of the image regions lying under the exposed regions of the filtering mask. For this purpose, we use a simple averaged gray-level morphological opening and closing filter $(3 \times 3)$. The opening filter in a sense extracts the lower bounding envelope of the ringing ripples, and in a dual manner the closing filter in a sense extracts the upper bounding envelope of the ringing ripples, and in their arithmetical average the ringing ripples are to a very great extent eliminated. All of these processings are performed through integer arithmetic and local min/max operations on gray-level data. Needless to say, the binary morphological operations of the previous steps are performed by logical shift, and AND/OR operations on binary data.

\section{Final image generation}

The final phase is the generation of the filter deringing output. For this purpose, we do the following. We keep the regions of the input image covered by the filtering mask intact. However, the regions of the input image exposed by the filtering mask (i.e., those regions which are filtered in the fourth phase) are copied from the output of the morphological smoothing filter and pasted on to the input image. This generates the output of the deringing filter.

We applied the deringing filter to Lena. Figure 9 shows the results for a compression ratio $100: 1$. It can be seen that the image after post-processing is much better in terms of perceptual performance than the reconstructed image in the middle.

\subsubsection{Post-processing using median filter}

This approach consists of two steps. First, an edge detection algorithm (Canny's algorithm) is used to determine the significant edges in a reconstructed image. Second, a median filter $(3 \times 3)$ is then applied to eliminate the ringing. A median filter is a nonlinear filter that chooses the median of 9 elements in a $3 \times 3$ window. The idea is to eliminate highamplitude noise without blurring the edges. Figure 10 shows the results. The perceptual performance did improve after post-processing. The perceptual performance improvement of median filtering is comparable to morphological filter described in Section 3.4.1 It appears that the median filter is simpler than the previous approach.

\subsection{New region-of-interest (ROI) enhancement capability}

In progressive image transmission, the most important information is transmitted first. The importance of pixels in a picture is reflected by the magnitude of its transformed coefficients. Therefore, the key idea here is that if we want to highlight a region in an image, we need to scale up the coefficients in that particular region. We achieve this goal by using Visual Basic. An interface of the software is shown in Figure 11. First, an image is loaded onto the screen. Second, a mouse is used to draw a box that one wants to highlight. The coordinates of the box are passed to the image algorithm so that the appropriate blocks will be highlighted. Third, a weight factor is selected from the screen. The weighting factor scales all the coefficients in the region of interest.

Figure 12 shows the performance of image compression with ROI enhancement. The tip of the gun barrel of a tank is highlighted. It can be seen that the image with ROI enhancement is better than the one without this option.

\subsection{Computational complexity analysis}

We have mainly used three methods in this research: DCT, wavelet, and GenLOT transforms. Since every component in coding and decoding is the same except in the transformation stage, we performed a complexity analysis of the three 


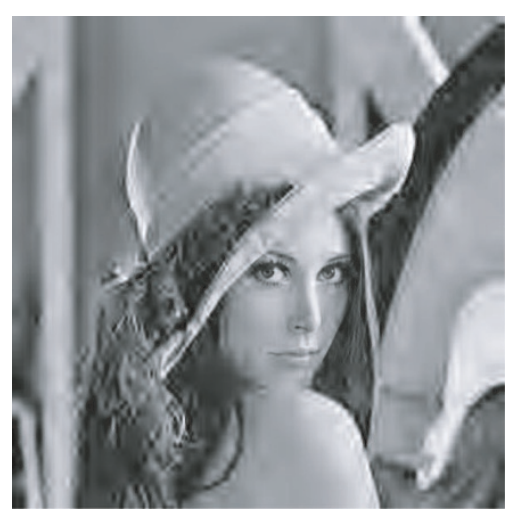

(a) $100: 1$ by OBTWC

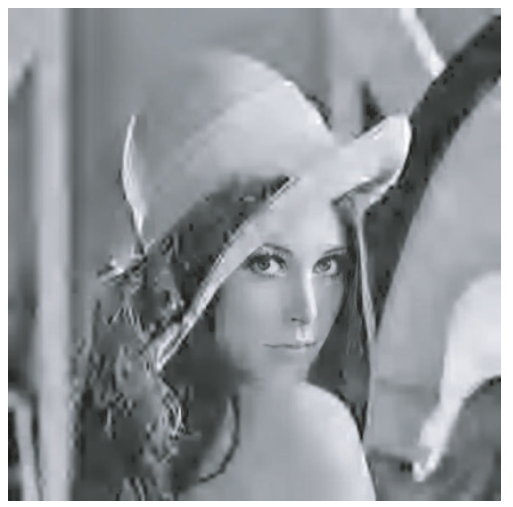

(b) $100: 1$ after post-processing

FIGURE 9: Effects of morphological deringing filter on Lena.

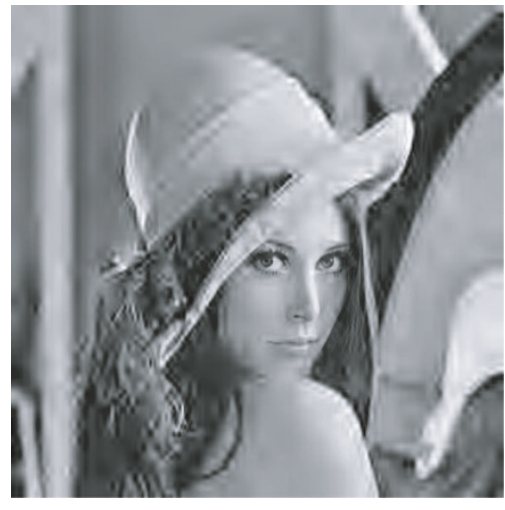

(a) $100: 1$

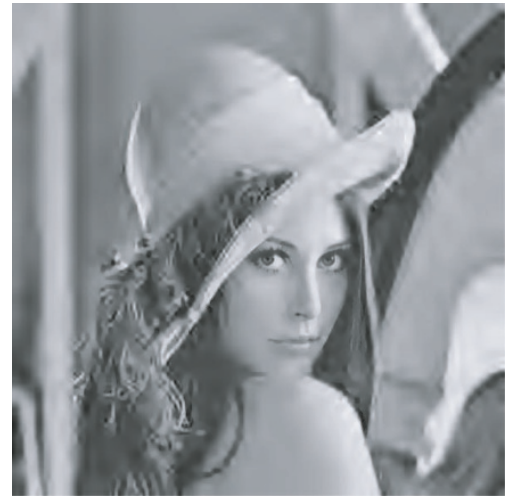

(b) $100: 1$ after post-processing

FIGURE 10: Post-processing using median filter.

schemes. Table 8 summarizes the number of computations by using software for a given $N \times N$ image. It is worth mentioning that if no parallel implementation for both DCT and GenLOT is done, then it can be seen that DCT is the most efficient one, followed by wavelet and GenLOT. Figure 13 shows the number of computations versus image size $N$. All three grow exponentially if no parallel implementation is used.

However, if one implements the DCT and GenLOT in a parallel manner by taking advantage of the block transformation characteristics, one can see that the DCT and GenLOT can be very efficient. As can be seen from Table 9 and Figure 14, DCT and GenLOT algorithms stay almost flat while the wavelet transform still grows exponentially.

\subsection{Summary of the results}

From all the experiments presented above, it is found that the proposed method can compress images with better or about the same PSNR as the two competing approaches. In all these examples, the visual quality of the compressed image from the proposed method is often better than the competing approaches. For those cases where the proposed method has slightly lower PSNR than the wavelet coder, there is little difference in visual quality. Also, the proposed post-processing techniques are found to be effective in removing the ringing artifacts at extreme compression ratio. In particular, the system supports selective enhancement of an ROI.

\section{CONCLUSIONS AND FURTHER RESEARCH}

In this paper, we presented a complete codec for image compression based on overlapped block transform, which has been tested extensively on benchmark images (Lena and Barbara), SAR, and color images. For aggressive image compression, post-processing is absolutely essential in order to reduce unavoidable coding artifacts. Thus, we also presented two methods that can enhance the perceptual quality of decompressed images. Finally, an ROI enhancement method is included in the proposed system, which can 


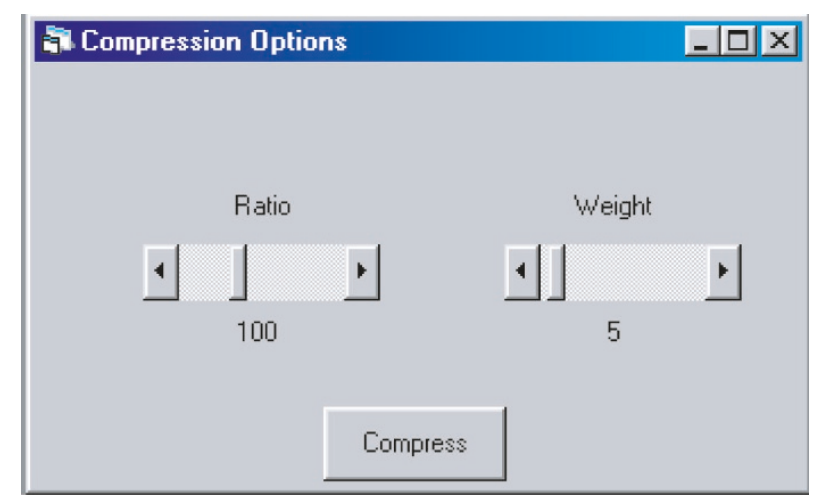

FiguRE 11: Interface of the region-of-interest program.

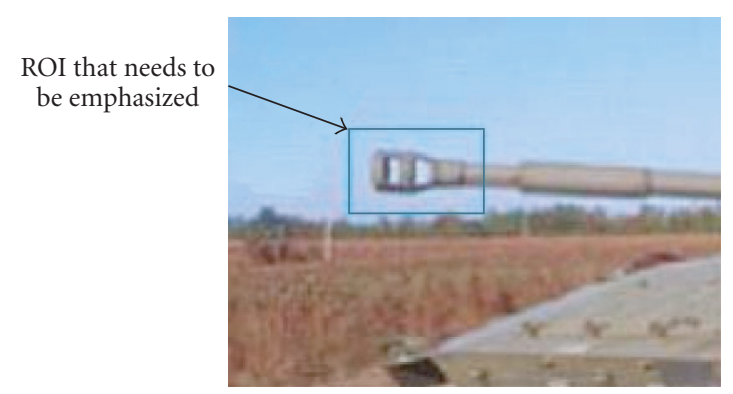

(a) Original image

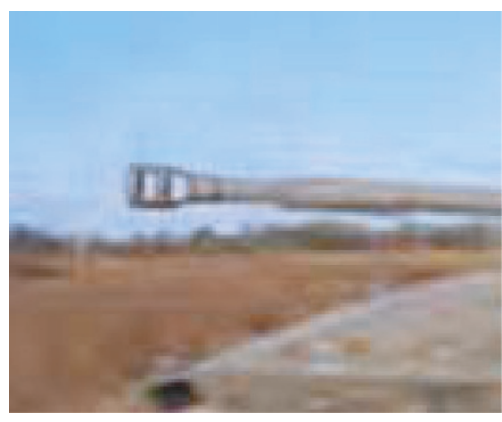

(b) $100: 1$ compression with enhanced tip of gun barrel

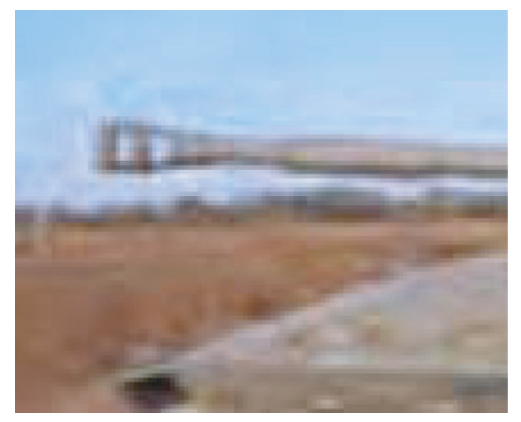

(c) $100: 1$ compression without ROI enhancement

FIGURE 12: Comparison of images with and without ROI enhancement.

control the compression ratio at certain critical regions of the images so that target recognition performance can be preserved. Extensive comparative studies with a commercial product (Summus - a wavelet-based codec), a JPEG baseline codec, and a JPEG-2000 codec showed that the proposed method achieved better performance in most cases.

While there exists extensive work on the reduction of blocking artifacts in a DCT-based scheme, such as the projection-onto-convex-sets (POCSs) approaches and others $[5,18-25]$, they are mostly post-processing techniques that work on a blocky image. Theoretically, since the information is already lost, these post-processing techniques cannot really reconstruct the original image but only improve the visual
TABLE 8: Software implementation: computational complexity of DCT, GenLOT, and wavelet for a given $N \times N$ image.

\begin{tabular}{l|cc}
\hline Method $\backslash$ complexity & Multiplications & Additions \\
\hline $8 * 8$ DCT & $3.25 N^{2}$ & $7.25 N^{2}$ \\
$8 * 40$ GenLOT & $40 N^{2}$ & $78 N^{2}$ \\
9/7 4-L wavelet & $11.9 N^{2}$ & $18.6 N^{2}$ \\
\hline
\end{tabular}

quality of the image by smoothing out the artifacts. The overlapped block transform solves the issue by virtually eliminating the block boundaries in the first place, and thus providing a more attractive way of addressing the blocking artifact 

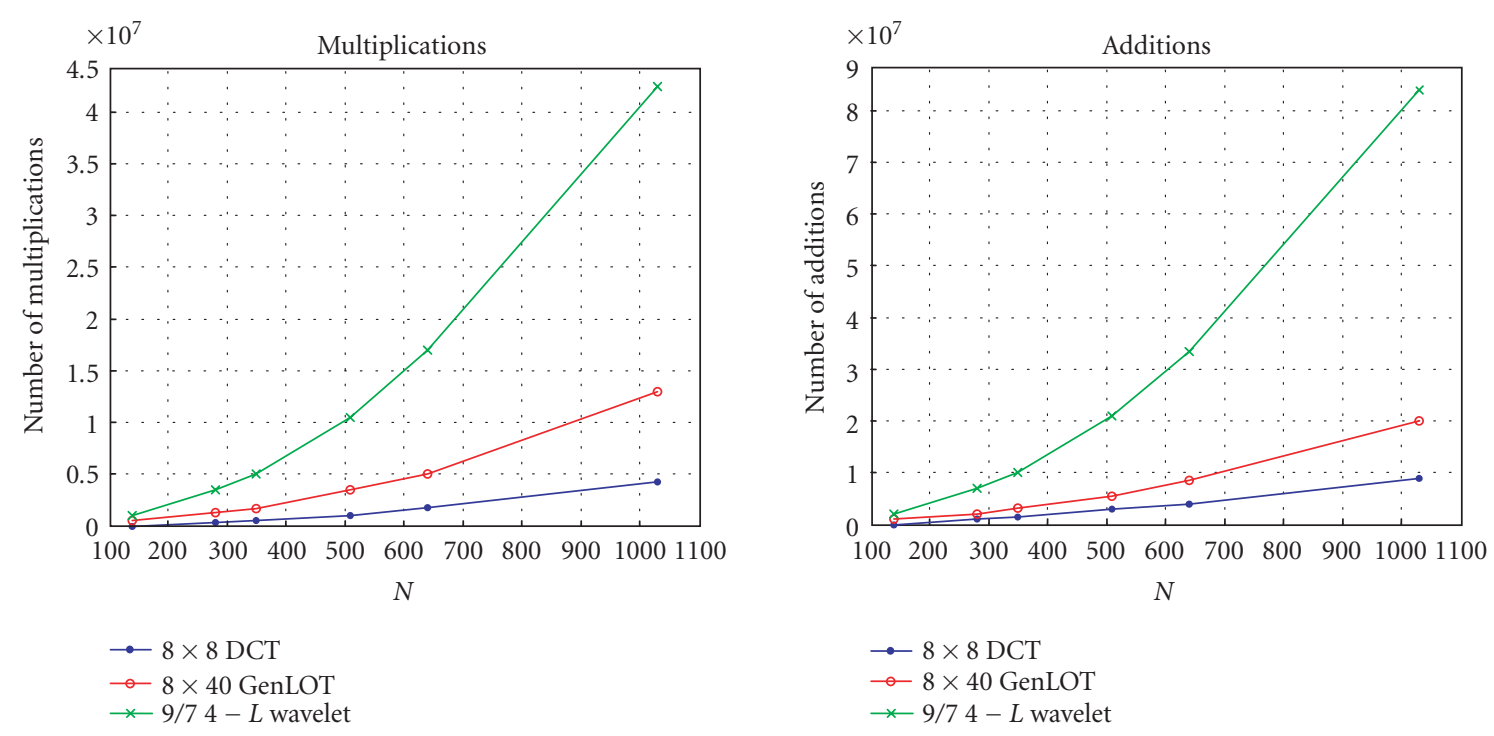

(b)

FIGURE 13: Software implementation. (All three grow exponentially with wavelet the least efficient.)

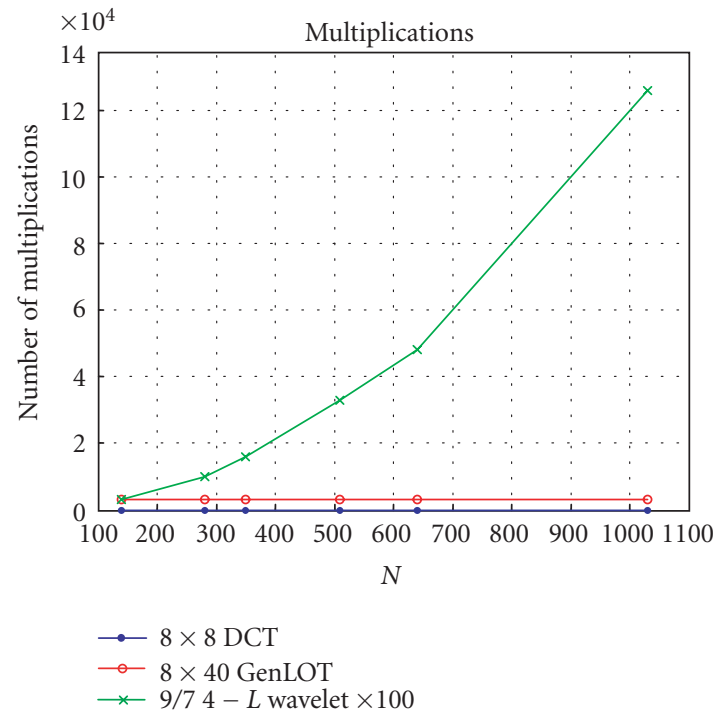

(a)

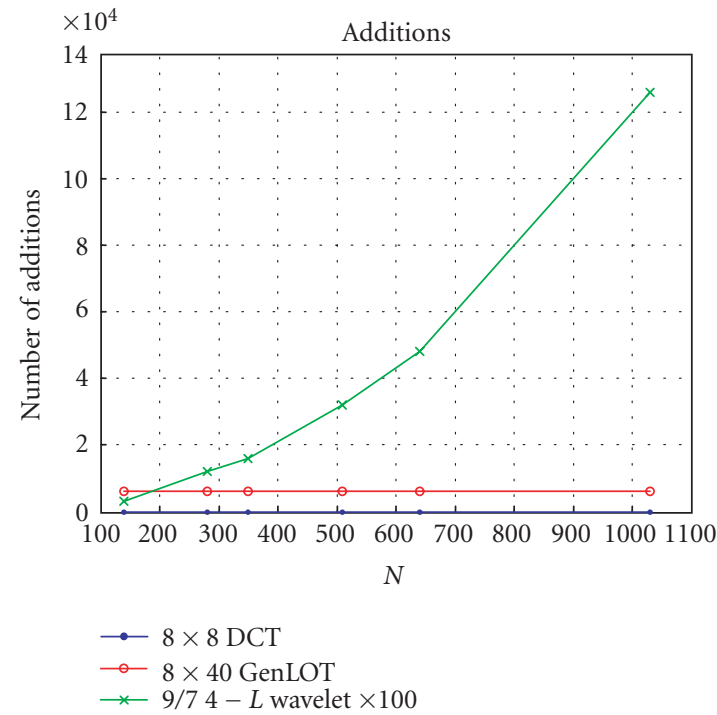

(b)

Figure 14: Parallel hardware implementation. (DCT and GenLOT stay almost flat; wavelet grows exponentially.)

issue. Nevertheless, it would be an interesting future task to compare such an overlapped transform approach with one leading deblocking algorithm to examine the performance of both approaches.

\section{ACKNOWLEDGMENTS}

This research was supported by the Ballistic Missile Defense Organization (BMDO) under Contract no. F33615-99-C1474 and managed by the Air Force. The encouragement
TABle 9: Parallel hardware implementation: computational complexity of DCT, GenLOT, and wavelet.

\begin{tabular}{l|cc}
\hline Method $\backslash$ complexity & Multiplications & Additions \\
\hline $8 * 8$ DCT & $3.25 \times 64$ & $7.25 \times 64$ \\
$8 * 40$ GenLOT & $40 \times 64$ & $78 \times 64$ \\
9/7 4-L wavelet & $11.9 N^{2}$ & $18.6 N^{2}$ \\
\hline
\end{tabular}

from Mr. Marvin Soraya at Wright-Patterson Air Force Laboratory is deeply appreciated. 


\section{REFERENCES}

[1] C. Kwan, B. Li, R. Xu, et al., "SAR image compression using wavelets," in Wavelet Applications VIII, vol. 4391 of Proceedings of SPIE, pp. 349-357, 2001.

[2] W. B. Pennebaker and J. L. Mitchell, JPEG: Still Image Compression Standard, Van Nostrand Reinhold, New York, NY, USA, 1993.

[3] H. S. Malvar, Signal Processing with Lapped Transforms, Artech House, Norwood, Mass, USA, 1992.

[4] H. S. Malvar, "Biorthogonal and nonuniform lapped transforms for transform coding with reduced blocking and ringing artifacts," IEEE Transactions on Signal Processing, vol. 46, pp. 1043-1053, 1998.

[5] H. C. Reeves and J. S. Lim, "Reduction of blocking effects in image coding," Optical Engineering, vol. 23, no. 1, pp. 34-37, 1984.

[6] R. L. de Queiroz, T. Nguyen, and K. R. Rao, "GenLOT: generalized linear-phase lapped orthogonal transform," IEEE Transactions on Signal Processing, vol. 44, no. 3, pp. 497-507, 1996.

[7] T. Tran and T. Nguyen, "On $M$-channel linear phase FIR filter banks and application in image compression," IEEE Transactions on Signal Processing, vol. 45, no. 9, pp. 2175-2187, 1997.

[8] Z. Xiong, K. Ramchandran, and M. T. Orchard, "Spacefrequency quantization for wavelet image coding," IEEE Transactions on Image Processing, vol. 6, no. 5, pp. 677-693, 1997.

[9] J. P. Princen, A. W. Johnson, and A. B. Bradley, "Subband/transform coding using filter bank designs based on time domain aliasing cancellation," in Proceedings of the IEEE International Conference on Acoustics, Speech and Signal Processing (ICASSP '87), pp. 2161-2164, Dallas, Tex, USA, April 1987.

[10] J. M. Shapiro, "Embedded image coding using zerotrees of wavelet coefficients," IEEE Transactions on Signal Processing, vol. 41, no. 12, pp. 3445-3462, 1993.

[11] A. Said and W. A. Pearlman, "A new fast and efficient image codec on set partitioning in hierarchical trees," IEEE Transactions on Circuits and Systems for Video Technology, vol. 6, pp. 243-250, 1996.

[12] "Compression with reversible embedded wavelets," RICOH Company Ltd. submission to ISO/IEC JTC1/SC29/WG1 for the JTC1.29.12 work item, 1995. Can be obtained on the World Wide Web, http://www.crc.ricoh.com/CREW.

[13] P. P. Vaidyanathan, Multirate Systems and Filter Banks, Prentice-Hall, Englewood Cliffs, NJ, USA, 1993.

[14] G. Strang and T. Nguyen, Wavelets and Filter Banks, WellesleyCambridge Press, Wellesley, Mass, USA, 1996.

[15] M. Vetterli and J. Kovačević, Wavelets and Subband Coding, Prentice-Hall, Englewood Cliffs, NJ, USA, 1995.

[16] R. A. DeVore, B. Jawerth, and B. J. Lucier, "Image compression through wavelet transform coding," IEEE Transactions on Information Theory, vol. 38, no. 2, part II, pp. 719-746, 1992.

[17] I. Daubechies, Ten Lectures on Wavelets, CBMS Conference Series, SIAM, Philadelphia, Pa, USA, 1992.

[18] N. F. Law and W. C. Siu, "Successive structural analysis using wavelet transform for blocking artifacts suppression," Signal Processing, vol. 81, no. 7, pp. 1373-1387, 2001.

[19] A. Zakhor, "Iterative procedures for reduction of blocking effects in transform image coding," IEEE Transactions on Circuits and Systems for Video Technology, vol. 2, no. 1, pp. 91-95, 1992.

[20] Y. Yang, N. P. Galatsanos, and A. K. Katsaggelos, "Regularized reconstruction to reduce blocking artifacts of block discrete cosine transform compressed images," IEEE Transactions on Circuits and Systems for Video Technology, vol. 3, no. 6, pp. 421-432, 1993.
[21] Y. Yang and N. P. Galatsanos, "Projection-based spatially adaptive reconstruction of block-transform compressed images," IEEE Transactions on Image Processing, vol. 4, no. 7, pp. 896908, 1995.

[22] S. D. Kim, J. Yi, H. M. Kim, and J. B. Ra, "A deblocking filter with two separate mode in block-based video coding," IEEE Transactions on Circuits and Systems for Video Technology, vol. 9, no. 2, pp. 156-160, 1999.

[23] H. W. Park and Y. L. Lee, "A post-processing method for reducing quantization effects in low bit-rate moving picture coding," IEEE Transactions on Circuits and Systems for Video Technology, vol. 9, no. 2, pp. 161-171, 1999.

[24] A. W.-C. Liew and H. Yan, "Blocking artifacts suppression in blockcoded images using overcomplete wavelet representation," IEEE Transactions on Circuits and Systems for Video Technology, vol. 14, no. 4, pp. 450-461, 2004.

[25] C. Weerasinghe, A. W.-C. Liew, and H. Yan, "Artifact reduction in compressed images based on region homogeneity constraints using the projections onto convex sets algorithm," IEEE Transactions on Circuits and Systems for Video Technology, vol. 12, no. 10, pp. 891-897, 2002.

C. Kwan received his B.S. degree in electronics with honors from the Chinese University of Hong Kong in 1988 and his M.S. and Ph.D. degrees in electrical engineering from the University of Texas at Arlington in 1989 and 1993, respectively. From April 1991 to February 1994, he worked in the Beam Instrumentation Department of the Superconducting Super Collider Laboratory (SSC) in Dallas, Tex, where he was heavily involved in the modeling, simulation, and design of modern digital controllers and signal processing algorithms for the beam control and synchronization system. He received an Invention Award for his work at SSC. Between March 1994 and June 1995, he joined the Automation and Robotics Research Institute in Fort Worth, where he applied neural networks and fuzzy logic to the control of power systems, robots, and motors. Since July 1995, he has been with Intelligent Automation, Inc. in Rockville, Md. He has served as the Principal Investigator/Program Manager for more than 65 different projects, with total funding exceeding 20 million dollars. Currently, he is the Vice President, leading research and development efforts in signal/image processing and controls. He has published more than 40 papers in archival journals and has had 100 additional refereed conference papers.

B. Li received a Ph.D. degree in electrical engineering from the University of Maryland, College Park, in 2000. He is currently an Assistant Professor of computer science and engineering in the Arizona State University. He was previously a Senior Researcher with Sharp Laboratories of America (SLA), Camas, Wash, working on multimedia analysis for consumer applications. He was the Technical Lead in developing Sharp's hiimpact technologies. He was also an adjunct faculty member with the Portland State University from 2003 to 2004. His research interests include pattern recognition, computer vision, statistic methods, and multimedia processing. He is a Senior Member of IEEE. 
R. Xu received the B.S. degree from Jiangsu University in 1982, and the M.S. degree in 1988 from Xi'an Jiaotong University, China, both in electrical engineering. From 1982 to 1985 and from 1988 to 1993, he was teaching at Jiangsu University as an Assistant Professor. From 1993 to 1994, he was a Visiting Scholar at Lehrstuhl für Allgemeine und Theoretische Elektrotechnik, Universität ErlangenNürnberg, Germany. Since 1994, he has been with Intelligent Automation, Inc. (IAI), USA, where he is currently a Principal Engineer. His research interests include array signal processing, image processing, fault diagnostics, network security, and control theory and applications. Over the last 11 years with IAI, he has worked on many different research projects in the above areas funded by various US government agencies such as DoD and NASA. He has also published over 20 journal and conference papers in the related areas.

X. Li received his B.S. and M.S. degrees in electrical engineering from Xi'an Jiaotong University, China, in 1992 and 1995, respectively. He obtained his Ph.D. degree in electrical engineering from the University of Cincinnati, Ohio, in 2004. From 1995 to 1999, he was an Assistant Professor at Xi'an Jiaotong University. He worked as a Visiting Researcher at Siemens Corporate Research (SCR), Princeton, NJ, in 2002, and Mitsubishi Electronic Research Labs (MERL), Cambridge, Mass, in 2003. Since 2004, he has been with Intelligent Automation, Inc. as a Research Engineer. His research interests include image/video processing and analysis, optical/electronic imaging, medical imaging, computer vision, machine learning, pattern recognition, artificial intelligence, real-time system, and data visualization. He is a Member of the IEEE, SPIE, and Sigma Xi.

T. Tran received the B.S. and M.S. degrees from the Massachusetts Institute of Technology, Cambridge, in 1993 and 1994, respectively, and the Ph.D. degree from the University of Wisconsin, Madison, in 1998, all in electrical engineering. In July of 1998, he joined the Department of Electrical and Computer Engineering, The Johns Hopkins University, Baltimore, Md, where he currently holds the rank of Associate Professor.

His research interests are in the field of digital signal processing, particularly in multirate systems, filter banks, transforms, wavelets, and their applications in signal analysis, compression, processing, and communications. He was the Codirector (with Professor J. L. Prince) of the 33rd Annual Conference on Information Sciences and Systems (CISS'99), Baltimore, Md, in March 1999. He received the NSF CAREER Award in 2001. In the summer of 2002, he was an ASEE/ONR Summer Faculty Research Fellow at the Naval Air Warfare Center Weapons Division (NAWCWD) at China Lake, Calif. He currently serves as an Associate Editor of the IEEE Transactions on Signal Processing as well as IEEE Transactions on Image Processing. He is also a Member of the Signal Processing Theory and Methods (SPTM) Technical Committee of the IEEE Signal Processing Society.
T. Nguyen received the B.S, M.S, and Ph.D. degrees in electrical engineering from the California Institute of Technology, Pasadena, in 1985, 1986, and 1989, respectively. He was with MIT Lincoln Laboratory from June 1989 to July 1994, as a member of the technical staff. During the academic year 1993-1994, he was a Visiting Lecturer at MIT and an Adjunct Professor at Northeastern University. From August 1994 to July 1998, he was with the Electrical and Computer Engineering (ECE) Department, University of Wisconsin, Madison. He was with Boston University from August 1996 to June 2001. He is currently a Professor at the ECE Department, University of California, San Diego (UCSD). His research interests include video processing algorithms and their efficient implementation. He is the coauthor (with Professor Gilbert Strang) of a popular textbook, Wavelets \& Filter Banks. He has over 200 publications. He received the NSF Career Award in 1995 and is currently the Series Editor (Digital Signal Processing) for Academic Press. He served as an Associate Editor for the IEEE Transaction on Signal Processing from 1994 to 1996, for the IEEE Transaction on Circuits and Systems from 1996 to 1997, and for the IEEE Transaction on Image Processing from 2004 to 2005 . He is a Fellow of the IEEE. 